\title{
Biomedical applications of gene editing
}

\author{
Pablo Perez-Pinera $^{1,2} \cdot$ Zheng-Yi Chen $^{3}$
}

Published online: 17 August 2016

(C) Springer-Verlag Berlin Heidelberg 2016

Long before Watson and Crick initially discovered the double helix structure of DNA, the very idea of manipulating human genes for therapeutic purposes existed as pure fantasy. Nevertheless, after entering clinical trials in 1990, gene therapy was no longer relegated to the fertile minds of science fiction writers alike. Having thoroughly captivated the interest of the global research community, it remains inarguable that the therapeutic delivery of DNA into human cells to correct genetic conditions has truly evolved into one of the most powerful developments throughout the history of medicine.

Despite early successes, many of the limitations of gene therapy soon became evident. Perhaps one of the most important unintended effects of introducing viral DNA into mammalian cells is the tumorigenic potential intrinsic to viral delivery methods. While many technical developments have been designed to overcome this obstacle, gene editing technologies seem to hold the most promise. Rather than randomly integrating DNA into the genome through the use of viral vectors, gene editing specifically aims to repair faulty genes with single base-pair resolution.

Pablo Perez-Pinera

pablo@illinois.edu

$\triangle$ Zheng-Yi Chen

Zheng-Yi_Chen@meei.harvard.edu

1 Department of Bioengineering, University of Illinois at Urbana-Champaign, 1270 Digital Computer Laboratory, MC-278, 1304 West Springfield Avenue, Urbana, IL 61801, USA

2 Institute for Genomic Biology, University of Illinois at Urbana-Champaign, Urbana, IL 61801, USA

3 Department of Otolaryngology, Harvard Medical School and Eaton-Peabody Laboratory, Massachusetts Eye and Ear Infirmary, Boston, MA 02114, USA
Most importantly, there are three distinct phases of technological advancement that characterize the gene editing revolution: zinc finger nucleases (ZFNs), transcription activator like effector nucleases (TALENs), and clustered regularly interspaced short palindromic repeats (CRISPRs). Although significant differences certainly exist in terms of efficiency, practicality, and cost, each of these technologies ultimately accomplishes the same objective - to introduce a targeted modification into the genome of living cells.

Still in their infancy, the impact of these technologies in advancing biomedicine and biomedical research has been remarkable. In fact, a retrospective analysis of published gene editing research reveals some interesting trends. The majority of publications reported using gene editing tools for either basic research purposes or for generating transgenic organisms (Fig. 1). During the past 2 years alone, we also have witnessed an important increase in the total number of publications that utilize gene editing techniques to engineer therapies targeting viral infections, to perform genetic screenings, and even to develop applications related to cancer research, whereas only $2.5 \%$ of these manuscripts actually focus on attempting to correct genetic diseases.

In this special issue of Human Genetics, based on these developments, we present a compendium of reviews to highlight some of the more notable aspects of gene editing with regard to improving human health.

\section{Correction of genetic diseases}

While the pathogeneses of most monogenic diseases have been known for several decades, detailed knowledge of their molecular and genetic bases has not yet resulted in cures; therefore, treatment options remain purely 
Fig. 1 Publication trends in genome editing. Searching the SCOPUS database for the terms [TITLE-ABS-KEY (crispr) OR TITLE-ABS-KEY (talen) OR TITLE-ABS-KEY (zfn) AND DOCTYPE (ar) AND PUBYEAR > 1995] revealed 3693 published research articles. These entries were further screened to remove redundancies and to classify the manuscripts according to major themes. The data were plotted using GraphPad Prism 7. "CRISPR Loci" includes publications that describe sequences of CRISPR loci in microorganisms but do not utilize CRISPRs for genome modification. This database search was performed on 06-12-2016

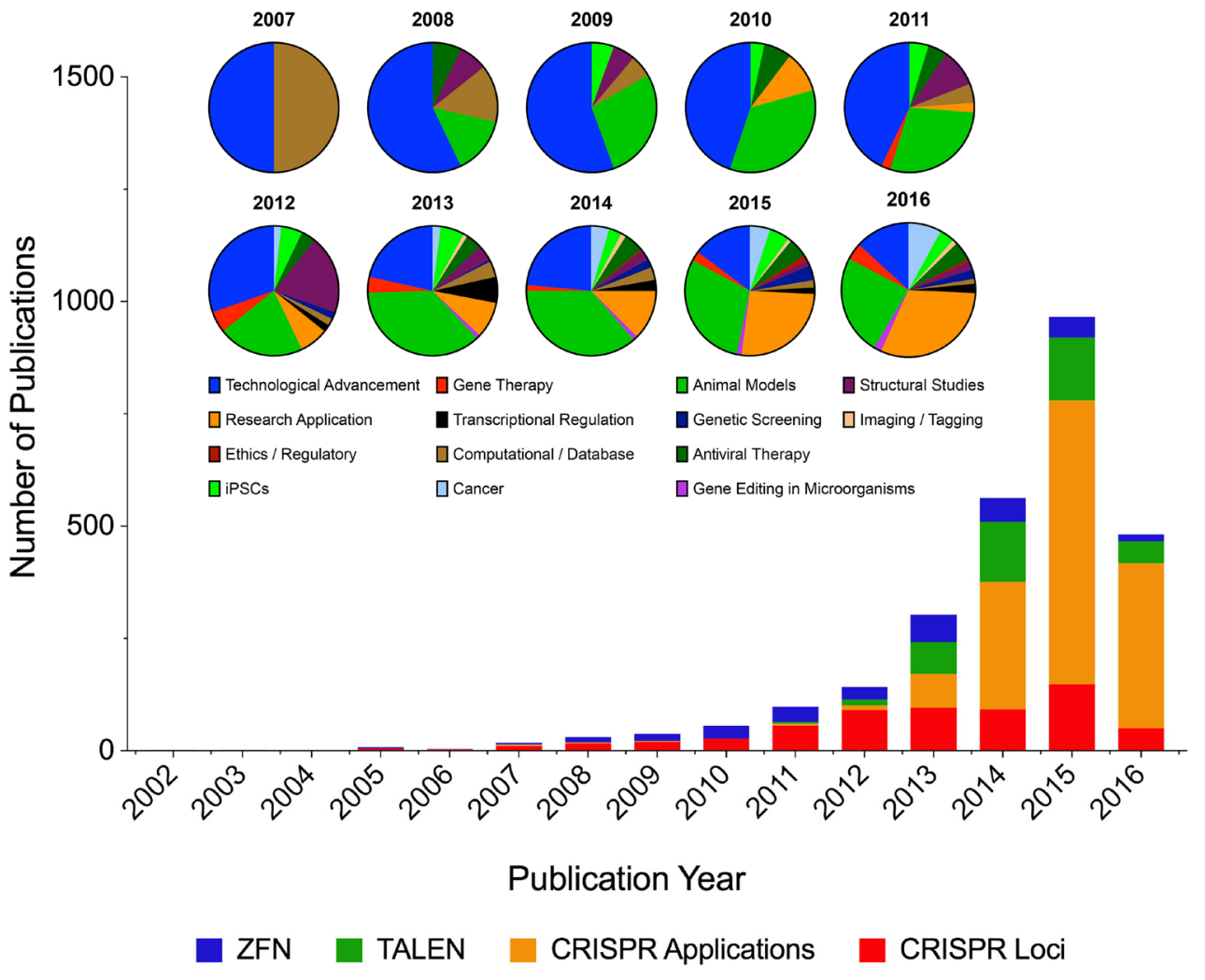

symptomatic in nature. However, gene editing empowers researchers to overcome some of the fundamental challenges inherent to the treatment of cystic fibrosis (Harrison et al. 2016), hemoglobinopathies (Cottle et al. 2016; Tasan et al. 2016), hemophilia (Park et al. 2016), and Duchenne muscular dystrophy (DMD) (Robinson-Hamm and Gersbach 2016).

\section{Gene editing of stem cells}

Induced pluripotent stem cells (iPSCs) hold great potential to become cell therapies for use in regenerative medicine; however, their very nature makes them resilient to transfection and DNA recombination. The review by Jang and Ye (2016) highlights the promises and challenges involved in rapidly generating functional and transplantable cell lineages from gene edited iPSCs in addition to describing some of the current issues related to their clinical translation.

\section{Antiviral therapies}

One of the most well-known applications of gene editing is the use of zinc finger nucleases to prevent HIV infection of T cells, which is currently in Phase II clinical trials. Herein, Benjamin et al. (2016) discuss the advantages of TALENs over both ZFN and CRISPRs in effectively fighting HIV. As further reviewed by Stone et al. (2016), the initial success of anti-HIV gene therapies paved the way for researchers to develop gene editing applications to eliminate or prevent viral infections such as $\mathrm{HCB}, \mathrm{HBV}$, EBV, HSV, HPV or HTLV.

\section{Cancer}

The genetic landscape of human cancers is enormously complex, with most tumors consisting of heterogeneous cell populations harboring multiple mutations. While next generation sequencing uncovers new mutations on a daily basis, understanding the mutations that have unique or causative roles still poses a formidable challenge. $\mathrm{Li}$ et al. (2016) discuss the importance of gene editing and how it simultaneously supports and accelerates cancer research.

\section{Forward/reverse genetics}

Gene editing has been used in both cells and animals to introduce mutations that recapitulate phenotypic changes associated with diseases. In conjunction with this reverse genetics approach, gene editing similarly enables forward genetics applications such as genome-scale functional 
screenings for identification of changes in DNA associated with a given phenotype. As such, Gurumurthy et al. (2016) review approaches based on forward and reverse genetics that improve our understanding of human disease.

\section{Emerging animal models of disease}

The decision to utilize mice as the preferred animal model for studying human disease is often contingent upon their lower cost and the relative ease with which their genes can be manipulated by homologous recombination. Unfortunately, in many instances - such as with cystic fibrosis, for example-mice are not good predictors of human disease. While pig models are superior with regard to anatomic and physiological characteristics, their genetic manipulation has been challenging. Genome editing technology has emerged as a powerful approach to make it possible to more effectively study diseases using pig models. Yao et al. (2016) provide a comprehensive review of the current technologies for creating transgenic pigs to model human diseases, thus likely foreshadowing the development of additional innovative applications and a wider range of treatment options.

In summary, biomedical research has experienced a profound transformation through the advent of gene editing, which has surpassed even our wildest expectations. This special issue of Human Genetics endeavors to present multiple insightful research efforts while highlighting fundamental gaps in technology that must be addressed to achieve the greatest potential that the genome editing revolution has to offer.

\section{References}

Benjamin R, Berges BK, Solis-Leal A, Igbinedion O, Strong CL, Schiller MR (2016) TALEN gene editing takes aim on HIV. Hum Genet. doi:10.1007/s00439-016-1678-2

Cottle RN, Lee CM, Bao G (2016) Treating hemoglobinopathies using gene-correction approaches: promises and challenges. Hum Genet. doi:10.1007/s00439-016-1696-0

Gurumurthy CB, Grati M, Ohtsuka M, Schilit SL, Quadros RM, Liu XZ (2016) CRISPR: a versatile tool for both forward and reverse genetics research. Hum Genet. doi:10.1007/s00439-016-1704-4

Harrison PT, Sanz DJ, Hollywood JA (2016) Impact of gene editing on the study of cystic fibrosis. Hum Genet. doi:10.1007/ s00439-016-1693-3

Jang YY, Ye Z (2016) Gene correction in patient-specific iPSCs for therapy development and disease modeling. Hum Genet. doi:10.1007/s00439-016-1691-5

Li X, Wu R, Ventura A (2016) The present and future of genome editing in cancer research. Hum Genet. doi:10.1007/ s00439-016-1713-3

Park CY, Lee DR, Sung JJ, Kim DW (2016) Genome-editing technologies for gene correction of hemophilia. Hum Genet. doi:10.1007/s00439-016-1699-x

Robinson-Hamm JN, Gersbach CA (2016) Gene therapies that restore dystrophin expression for the treatment of Duchenne muscular dystrophy. Hum Genet. doi:10.1007/s00439-016-1725-z

Stone D, Niyonzima N, Jerome KR (2016) Genome editing and the next generation of antiviral therapy. Hum Genet. doi:10.1007/ s00439-016-1686-2

Tasan I, Jain S, Zhao H (2016) Use of genome-editing tools to treat sickle cell disease. Hum Genet. doi:10.1007/s00439-016-1688-0

Yao J, Huang J, Zhao J (2016) Genome editing revolutionize the creation of genetically modified pigs for modeling human diseases. Hum Genet. doi:10.1007/s00439-016-1710-6 